\title{
Fabrication of large-pore mesoporous Ca-Si-based bioceramics for bone regeneration
}

\author{
This article was published in the following Dove Press journal: \\ International Journal of Nanomedicine \\ 15 November 2017 \\ Number of times this article has been viewed
}

\author{
Deliang Zeng ${ }^{1,2}$ \\ Xingdi Zhang ${ }^{3}$ \\ Xiao Wang ${ }^{1,2}$ \\ Lingyan Cao' \\ Ao Zheng ${ }^{1,2}$ \\ Jiahui $\mathrm{Du}{ }^{1,2}$ \\ Yongsheng $\mathrm{Li}^{3}$ \\ Qingfeng Huang' \\ Xinquan Jiang ${ }^{1,2}$
}

'Department of Prosthodontics, School of Medicine, Ninth People's Hospital affiliated to Shanghai Jiao Tong University, Shanghai, People's Republic of China; ${ }^{2}$ Oral Bioengineering Laboratory, Shanghai Research Institute of Stomatology, School of Medicine, Ninth People's Hospital Affiliated to Shanghai Jiao Tong University, Shanghai, People's Republic of China; ${ }^{3}$ Laboratory of Low-Dimensional Materials Chemistry, Key Laboratory for Ultrafine Materials of Ministry of Education, School of Materials Science and Engineering, East China University of Science and Technology, Shanghai, People's Republic of China
Correspondence: Xinquan Jiang Department of Prosthodontics, Ninth People's Hospital affiliated to Shanghai Jiao Tong University, School of Medicine, Zhizaoju Road, Shanghai 2000II, People's Republic of China

Tel +8621 63I354II2

Fax +86 21 63136856

Email xinquanj@aliyun.com

\begin{abstract}
Our previous study revealed that mesoporous Ca-Si-based materials exhibited excellent osteoconduction because dissolved ions could form a layer of hydroxycarbonate apatite on the surface of the materials. However, the biological mechanisms underlying bone regeneration were largely unknown. The main aim of this study was to evaluate the osteogenic ability of large-pore mesoporous Ca-Si-based bioceramics (LPMSCs) by alkaline phosphatase assay, real-time PCR analysis, von Kossa, and alizarin red assay. Compared with large-pore mesoporous silica (LPMS), LPMSCs had a better effect on the osteogenic differentiation of dental pulp cells. LPMSC-2 and LPMSC-3 with higher calcium possessed better osteogenic abilities than LPMSC-1, which may be related to the calcium-sensing receptor pathway. Furthermore, the loading capacity for recombinant human platelet-derived growth factor-BB was satisfactory in LPMSCs. In vivo, the areas of new bone formation in the calvarial defect repair were increased in the LPMSC-2 and LPMSC-3 groups compared with the LPMSC-1 and LPMS groups. We concluded that LPMSC-2 and LPMSC-3 possessed both excellent osteogenic abilities and satisfactory loading capacities, which may be attributed to their moderate $\mathrm{Ca} / \mathrm{Si}$ molar ratio. Therefore, LPMSCs with moderate $\mathrm{Ca} / \mathrm{Si}$ molar ratio might be potential alterative grafts for craniomaxillofacial bone regeneration.
\end{abstract}

Keywords: mesoporous Ca-Si-based materials, dental pulp cells, rat calvarial defect

\section{Introduction}

Since the invention of Mobil Composition of Matter No 41 in 1992, mesoporous silica (MS) materials have experienced a rapid development and are now widely used in biomedical areas, the adsorption and separation of proteins, catalysis, and so on. ${ }^{1-3}$ Studies have shown that proteins absorbed in the mesoporous materials could improve the thermal stability of proteins; ${ }^{4}$ therefore, MS has a great potential in the protein loading field. The growth factor, a kind of therapeutic protein, has a strong osteoinductive ability so that it can effectively promote bone tissue regeneration. ${ }^{5,6}$ The common growth factors, including platelet-derived growth factor (PDGF), ${ }^{7}$ were applied in the clinical treatment of bone repair. These growth factors have a high molecular weight and a large molecular size (usually $>10 \mathrm{~nm}$ ). ${ }^{8-10} \mathrm{Up}$ to now, the pore diameters of mesoporous materials are $<7 \mathrm{~nm}$; thus, they cannot effectively load large molecular proteins, and their application in bone regeneration is severely affected.

At present, the common method for synthesizing large-pore MS is adding organic solvents (such as 1,3,5-trimethylbenzene) as pore-expanding agents. ${ }^{11}$ Although the MS synthesized with this method has a large pore size and high pore volume, the addition of organic solvents complicates the process. Organic and inorganic complexes were formed by silicic acid species and an organic template in most MS synthesis processes, and then, the organic template was removed to get the corresponding 
mesoporous structure. High-temperature calcination ${ }^{12}$ and organic solvent extraction ${ }^{13}$ are two common methods to remove organic templates. Although high-temperature calcination could completely remove organic templates, a large number of $\mathrm{Si}-\mathrm{OH}$ groups in the inner and outer surfaces of the materials would also disappear at the same time. The presence of the $\mathrm{Si}-\mathrm{OH}$ groups not only enhances the hydrophilicity of MS, but also makes it easier for MS to become functional; it plays an important role in the application of MS. Organic solvent extraction at low temperatures $\left(<100^{\circ} \mathrm{C}\right)$ can avoid the loss of $\mathrm{Si}-\mathrm{OH}$, but organic templates cannot be removed completely, even after many attempts at extraction. Currently, there is no single way to synthesize MS without organic templates.

MS has excellent biocompatibility, a modifiable surface, and an adjustable pore size, but it lacks bone bioactivity. In 1971, Professor Hench prepared calcium Si-based bioactive glass, ${ }^{14-16}$ which not only has good biocompatibility, but also has biological activity. This kind of material forms a layer of hydroxyapatite on the surface of the material after being implanted in the body. As we all know, hydroxyapatite is one of the main components of bone; therefore, the material can bond with bone tissue. In addition, changes in the calcium concentration have important links with the cell cycle. During the absence of extracellular calcium, the cell cycle stagnates at stage G1. If the concentration of calcium is raised at this time, the cells can then continue to conduct DNA synthesis, that is, to promote cell proliferation. ${ }^{17,18}$ Moreover, calcium is a common allosteric growth factor that regulates cell differentiation by the cytoplasm, organelles, and enzymes in the nucleus. ${ }^{19,20}$ Although elevated calcium ions inhibit the early osteogenic differentiation of cells, they can promote the maturation and mineralization of cells at the later stage, which is beneficial to the formation of bone. ${ }^{21}$ However, this may lead to apoptosis when the extracellular calcium concentration is too high. Therefore, the appropriate calcium content is closely linked to the biological activity of bone scaffold materials.

In this study, we first synthesized large-pore MS (LPMS) as the template and silicon source and then doped LPMS with $\mathrm{Ca}\left(\mathrm{NO}_{3}\right)_{2} \cdot 4 \mathrm{H}_{2} \mathrm{O}$ to synthesize large-pore Ca-Si-based bioceramics (LPMSCs). To assess the osteoproduction of the material, the effects of LPMSCs on dental pulp cells (DPCs) were evaluated by using analyses of MTT, alkaline phosphatase (ALP), von Kossa, alizarin red S (ARS), and real-time PCR. Meanwhile, the CaSR pathway inhibitor (NPS2143) was utilized to explore the possible mechanism of material-promoting osteogenesis. The rat calvarial defect model was selected, and the LPMSCs were implanted into the defect for 8 weeks. Then, the areas of new bone formation were determined by histological observation to assess the bone regeneration ability of the LPMSCs.

\section{Materials and methods Preparation and characterization of LPMSCs}

LPMS was prepared by using a simple one-step method as described previously. ${ }^{22}$ In contrast, the sample was calcined at $550^{\circ} \mathrm{C}$ for 6 hours and named LPMS-cal. Then, LPMSCs were synthesized via a solid-state reaction by combining LPMS and $\mathrm{Ca}\left(\mathrm{NO}_{3}\right)_{2} \cdot 4 \mathrm{H}_{2} \mathrm{O}$. By changing the amount of $\mathrm{Ca}\left(\mathrm{NO}_{3}\right)_{2} \cdot 4 \mathrm{H}_{2} \mathrm{O}$, the LPMSCs with different $\mathrm{Si} / \mathrm{Ca}$ molar ratios were synthesized and named LPMSC-1 $(\mathrm{Si} / \mathrm{Ca}=4: 1)$, LPMSC-2 ( $\mathrm{Si} / \mathrm{Ca}=2: 1)$, and LPMSC-3 ( $\mathrm{Si} / \mathrm{Ca}=1: 1)$.

The structure and composition of our scaffolds were tested by scanning electron microscopy (SEM), wide-angle $\mathrm{X}$-ray diffraction, small-angle X-ray Raman scattering (SXRS), thermogravimetric (TG), high-resolution X-ray photoelectron spectroscopy (XPS) spectra, Barrett-JoynerHalenda (BJH) analysis, Fourier transform infrared (FT-IR) spectrometry, and energy-dispersive spectrometry (EDS).

\section{DPCs isolation and culture}

All animals were obtained from the Ninth People's Hospital Animal Center (Shanghai, People's Republic of China). All the experiments followed the guidelines of Animal Experimental Ethical Inspection-Shanghai Ninth People's Hospital affiliated to Shanghai Jiao Tong University, School of Medicine (No HKDL[2016]132). Briefly, after euthanizing the male Sprague-Dawley rats (4 weeks old) with an overdose of pentobarbital injected intraperitoneally, the pulp tissue was pulled out of the molars. Then, the tissue was immersed in PBS and digested in pancreatin for 5 minutes and collagenase type I for 1 hour. Next, we centrifuged the cell suspension and cultured the cells with DMEM (Gibco ${ }^{\circledR}$; Thermo Fisher Scientific, Waltham, MA, USA) with 10\% fetal bovine serum. After reaching a confluence of $\sim 80 \%$, the cells were passaged in fresh DMEM.

DPCs were digested from culture dishes, resuspended in the DMEM, and then seeded on the LPMS or LPMSCs. After 1 day, the complexes were fixed and then observed by SEM or stained by Calcein AM $(2 \mu \mathrm{m})$, which was used to observe live cells on the scaffolds.

\section{Cell metabolic activity}

Cell metabolic activity was assessed by using the MTT assay. Briefly, DPCs seeded on the scaffolds ( $10^{4}$ cells/disk) were incubated in 12-well plates for 1, 3, and 5 days. ${ }^{23}$ Each 
sample was incubated with MTT solution at $37^{\circ} \mathrm{C}$ for 4 hours in an incubator. Then, the reaction was stopped by DMSO, and the absorbance was read at $490 \mathrm{~nm}$ with a microplate reader (Elx800; BioTek Instruments, Winooski, VT, USA). DPCs cultured in medium without the scaffold were used as the controls. The results were reflected in OD absorbance value units.

\section{Cell differentiation assay}

To determine the differentiation of DPCs stimulated by the scaffolds, the cells were seeded onto scaffolds $\left(4 \times 10^{4}\right.$ cells/disk) or cultured in extracts. The LPMS and LPMSC extracts were prepared according to the methods (ISO10993-1) as in our previous study. ${ }^{23}$ ALP staining and activity were measured as previously described. ${ }^{24}$ ALP staining was investigated according to the instructions of the kit (Beyotime, Haimen, People's Republic of China). The ALP activity of DPCs seeded onto LPMS and LPMSCs was quantified by utilizing p-nitrophenylphosphate (SigmaAldrich Co., St Louis, MO, USA). DPCs cultured in medium without the scaffold were used as the controls. To detect mineralization, DPCs were seeded onto scaffolds or cultured in extracts for 28 days. Then, the cells were stained with $40 \mathrm{mM}$ ARS solution or $1 \% \mathrm{AgNO}_{3}$ after being fixed in $70 \%$ ethanol for 1 hour.

After the DPCs were seeded onto scaffolds or cultured in extracts with or without the CaSR signaling pathway inhibitor NPS2143 for 7 days, real-time PCR was conducted to assess gene expression. The RNA was extracted using the TRIzol ${ }^{\circledR}$ reagent (Invitrogen; Thermo Fisher Scientific), and cDNA was synthesized from the RNA using the cDNA synthesis kit (TaKaRa, Tokyo, Japan). The gene expression was assessed by the SYBR Premix Ex Taq ${ }^{\circledR}$ kit (TaKaRa) and Bio-Rad real-time PCR system (Bio-Rad Laboratories Inc., Hercules, CA, USA). The assessed markers were the Runx2, Osterix, Ocn, Vegf, Ppary, Dmp-1, Dspp, Bmp-2, CaSR, Opg, and Rankl; GAPDH was used as the housekeeping gene for normalization.

\section{Release of recombinant human PDGF-BB (rhPDGF-BB) from scaffolds}

The porous LPMS and LPMSC scaffolds ( $5 \mathrm{~mm}$ in diameter and $2 \mathrm{~mm}$ thick) were impregnated with rhPDGF-BB (0.3 mg/mL; Osteohealth Company, Shirley, NY, USA), which has received the US Food and Drug Administration certification. The composite was then freeze-dried to obtain a factor release system. To determine the release rate of rhPDGF-BB from LPMS and LPMSCs, the scaffolds were soaked in $\mathrm{PBS}$ at $37^{\circ} \mathrm{C} .{ }^{25}$ Then, $100 \mu \mathrm{L}$ of supernatant was collected at $0,6,12,24,48,96$, and 192 hours and replaced by an equal volume of PBS. The concentration of rhPDGF-BB was tested by an enzyme-linked immunosorbent assay (R\&D Systems, Inc., Minneapolis, MN, USA) according to the manufacturer's instructions.

\section{Repair of calvarial defect in rats}

As described in our previous study, ${ }^{25}$ two circular defects ( $5 \mathrm{~mm}$ in diameter) were created in both sides of rat calvarial bone after anesthesia. The defects were randomly filled with the following implants: LPMS, LPMSC-1, LPMSC-2, and LPMSC-3 alone. Then, the incision was sterilized and sutured, and the antibiotics (streptomycin and penicillin) were administered subcutaneously for the prevention of infection. Finally, the animals were sacrificed at week 8 .

\section{Histological and histomorphometric observations}

Each sample was cut into two blocks along the cranial raphe. One-half of the blocks were decalcified and sliced for H\&E staining. The un-decalcified samples were stained with van Gieson's picro-fuchsin as described in our previous study. ${ }^{23}$ Then, the areas of newly formed bone were analyzed by Image-Pro Plus system. Three randomly selected sections from the serial sections collected from each sample were analyzed. The percentages of newly formed bone area in all calvarial defect sites were calculated by longitudinal sections. The organs, including the liver, spleen, kidneys, lungs, and heart, were stained with $H \& E$ to analyze their pathological status.

\section{Statistical analysis}

In this study, the SAS 8.2 statistical software package (SAS Institute Inc., Cary, NC, USA) was used for all statistical analyses with one-way analysis of variance and the StudentNewman-Keuls post hoc or Kruskal-Wallis nonparametric procedure followed by the Mann-Whitney $U$-test. In addition, Bonferroni correction should be applied after the Mann-Whitney $U$-test followed by Kruskal-Wallis test to prevent type I error. $p<0.05$ is considered statistically significant.

\section{Results \\ Characterization of LPMS and LPMSCs}

This simple one-step method successfully synthesized differently shaped scaffolds and the interconnected macroporous structure of LPMSCs (Figure 1A-D). The mesoporous structure of the LPMS surface can be clearly observed by SEM (Figure 1E), and a component of the LPMS scaffold 


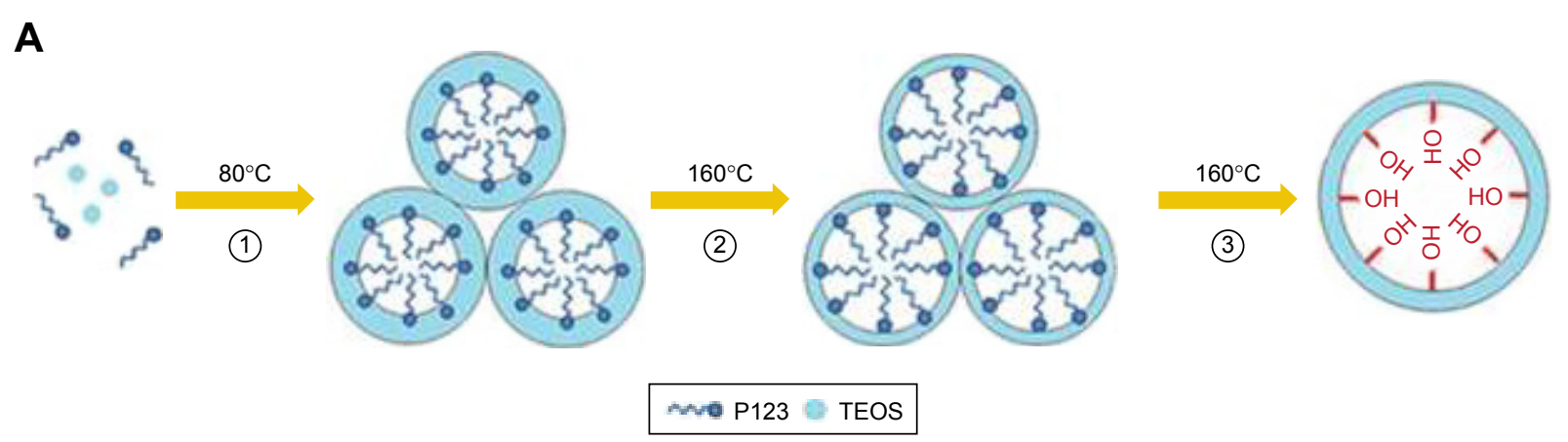

B

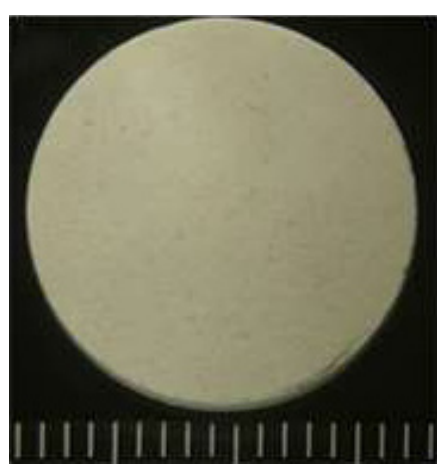

E

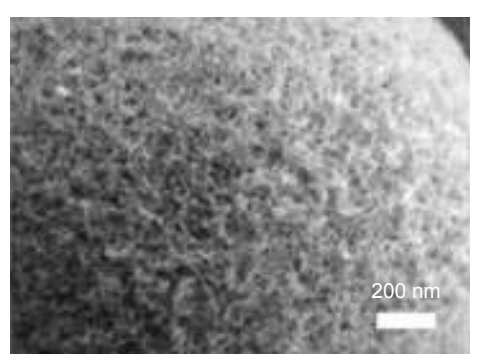

H

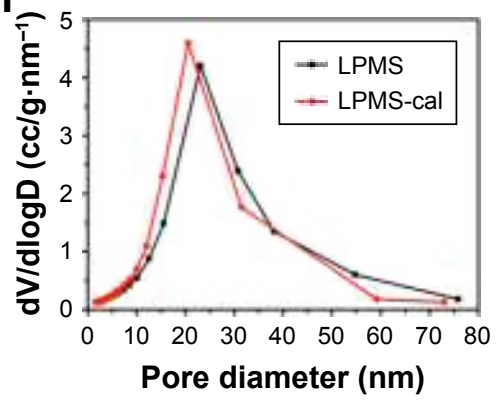

C

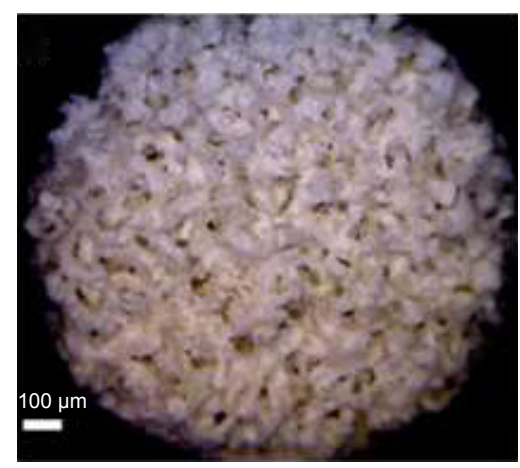

$\mathbf{F}$

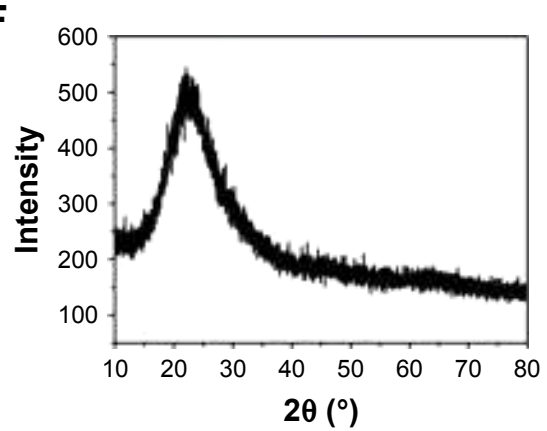

I

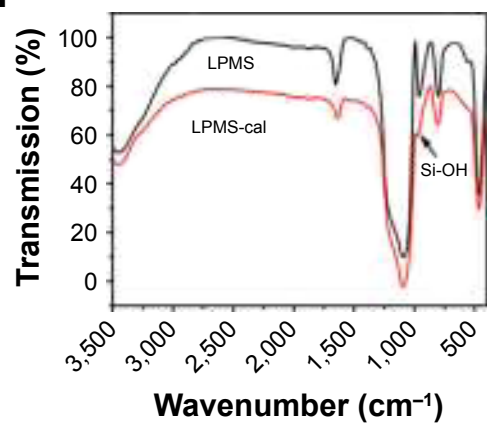

D

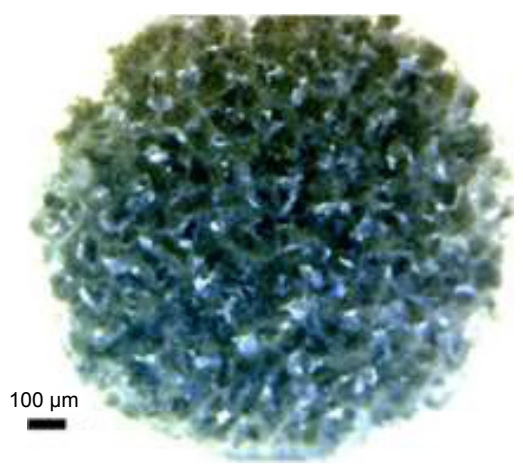

G

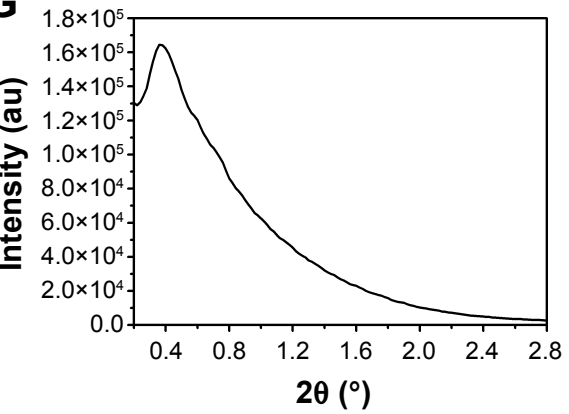

J

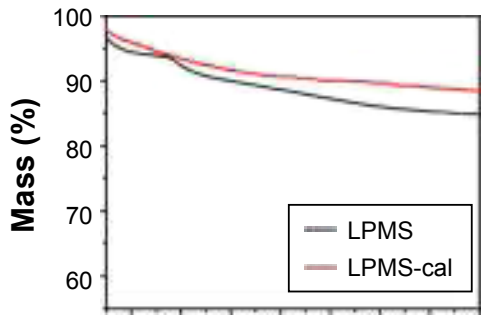

100200300400500600700800

Temperature $\left({ }^{\circ} \mathrm{C}\right)$

Figure I The characterization of LPMS and LPMSC scaffolds. The proposed mechanism for the preparation of LPMS (A). Photograph of LPMSC with different shapes (B and C). Reverse color photograph of LPMSC (D). Scanning electron microscopy image of LPMSC (E). Wide-angle XRD pattern of LPMS (F). Small-angle XRS patterns of LPMS (G). Pore size distribution curves of LPMS and LPMS-cal (H). FT-IR spectrum of LPMS and LPMS-cal (I). TG curves of LPMS and LPMS-cal (J).

Abbreviations: FT-IR, Fourier transform infrared; LPMS, large-pore mesoporous silica; LPMSC, large-pore mesoporous Ca-Si-based bioceramics; TEOS, tetraethyl orthosilicate; TG, thermogravimetric; XRD, X-ray diffraction; XRS, X-ray Raman scattering.

was a typical indefinite form of $\mathrm{SiO}_{2}$ (Figure 1F). The SXRS spectrum indicated that there were obvious scattering peaks at $\sim 0.4^{\circ}$ (Figure $1 \mathrm{G}$ ). According to the principle of the Prague equation, the diffraction peak moves to a small angle when the pore size of the material increases. Thus, the pore structure of LPMS was further proved. Figure $1 \mathrm{H}$ shows a $\mathrm{BJH}$ pore size distribution calculated according to the adsorption branch. It could be seen that the pore size of LPMS-cal 
(20.3 nm) was slightly smaller than the pore diameter of LPMS (22.8 nm). We only observed the characteristic peaks of Si-O-Si $\left(1,085,800\right.$, and $\left.470 \mathrm{~cm}^{-1}\right)$ and $\mathrm{Si}-\mathrm{OH}\left(960 \mathrm{~cm}^{-1}\right.$; Figure 1I) and did not observe the characteristic peaks of $\mathrm{C}-\mathrm{H}$, which demonstrated that the samples were free of organic templates. Moreover, the TG curves indicated that the weight losses of LPMS and LPMS-cal at $180^{\circ} \mathrm{C}$ were only $7.2 \%$ and $4.1 \%$ (Figure $1 \mathrm{~J}$ ), far below the loss of the conventional MS ( $\sim 50 \%)$, which suggested that there was no organic template in our scaffolds.

Figure 2A is the XPS full spectrum of the sample. Only $\mathrm{O}$ and $\mathrm{Si}$ were present in the LPMS, whereas $\mathrm{Ca}$ was present in the LPMSCs (LPMSC-1, LPMSC-2, and LPMSC-3). The peak was raised with increasing $\mathrm{Ca}$ content, which was consistent with the EDS results (Figure 2B-D). Moreover, the pore sizes of LPMSC-1 (15.7 nm), LPMSC-2 (15.8 nm), and LPMSC-3 $(21.3 \mathrm{~nm})$ were smaller than that of the LPMS due to the addition of $\mathrm{Ca}$. As shown in Figure 2E-L, adherent DPCs were observed on the four materials, and no significant difference in morphology and structure was observed, which suggested that our scaffolds were not clearly toxic and suitable for the following biology experiment.

A

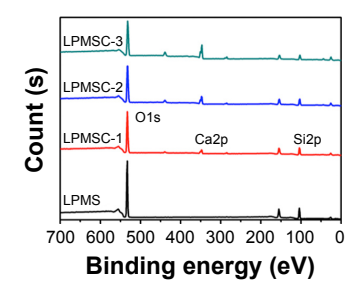

LPMS
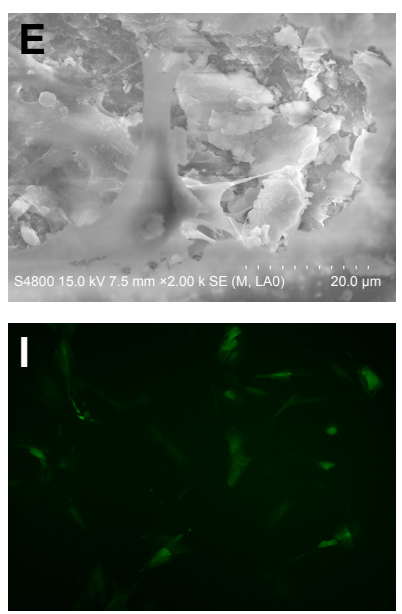

B

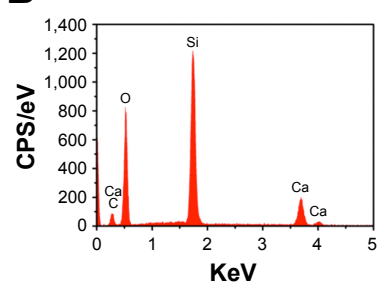

LPMSC-1
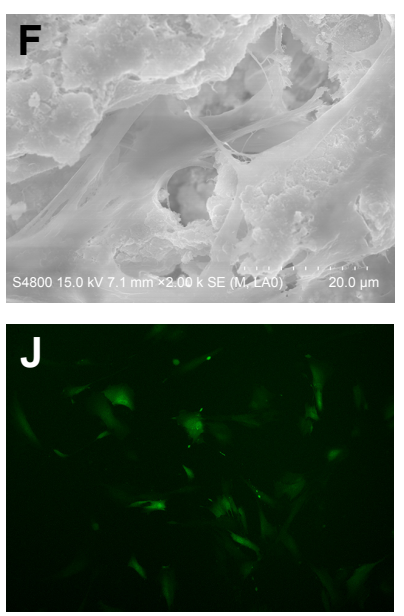

Metabolic activity and osteogenic differentiation of DPCs seeded on scaffolds

The MTT results showed no significant differences among the five groups on days 1 and 3 (Figure 3A). However, the metabolic activity of DPCs cultured in medium proliferated was weaker than that of the cells seeded onto the LPMS and LPMSCs at day $5(p<0.05)$. LPMSC-2 was superior to LPMS in promoting cell metabolic activity on day 5 , which may be linked to the addition of $\mathrm{Ca}$.

ALP staining, von Kossa staining, ARS staining, and real-time PCR were adopted to assess the effect of LPMS and LPMSCs on DPSC differentiation. After 7 days of culture, ALP staining of the DPCs seeded onto the LPMSCs was more intense than that of LPMS group (Figure 3B). In addition, the trend of von Kossa staining and ARS staining was similar to that of ALP staining. The real-time PCR results showed increased gene expression (Runx2, Osterix, Ocn, and Dmp-1) for cells seeded on the LPMSCs (Figure 3C). Runx 2 and Osterix expressions were significantly enhanced in the DPCs seeded onto the LPMSCs compared with the cells on the LPMS scaffolds $(p<0.05)$. Moreover, the Vegf

C

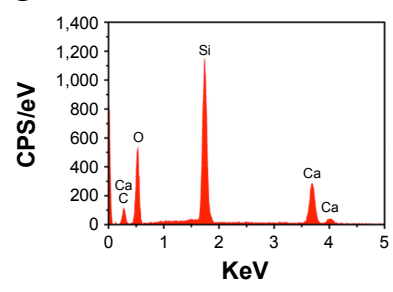

D

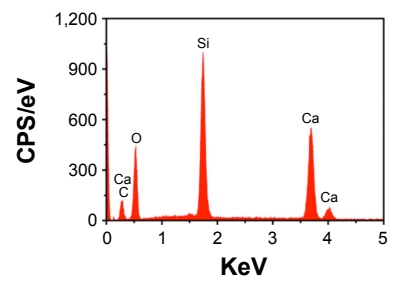

LPMSC-2

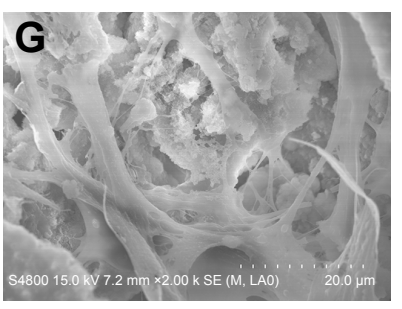

LPMSC-3
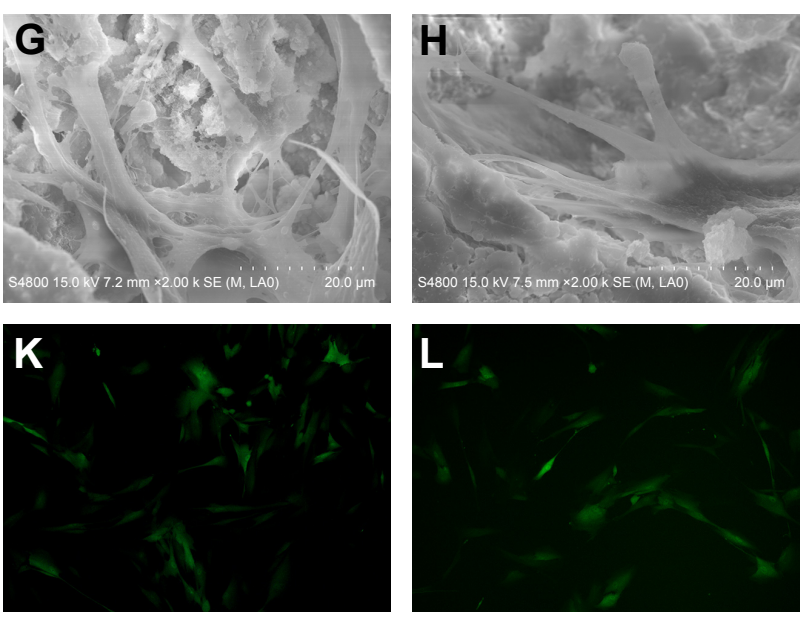

Figure 2 The characterization and cytotoxicity of LPMSCs. XPS full spectrum of different samples (A). EDS analysis for LPMSC-I (B), LPMSC-2 (C), and LPMSC-3 (D). SEM $(\mathbf{E}-\mathbf{H})$ and fluorescence $(\mathbf{I}-\mathbf{L})$ images of DPCs on different samples after culturing for 48 hours.

Abbreviations: CPS, counts per second; DPCs, dental pulp cells; LPMSC, large-pore mesoporous Ca-Si-based bioceramics; EDS, energy-dispersive spectrometry; SEM, scanning electron microscopy; XPS, X-ray photoelectron spectroscopy. 
A
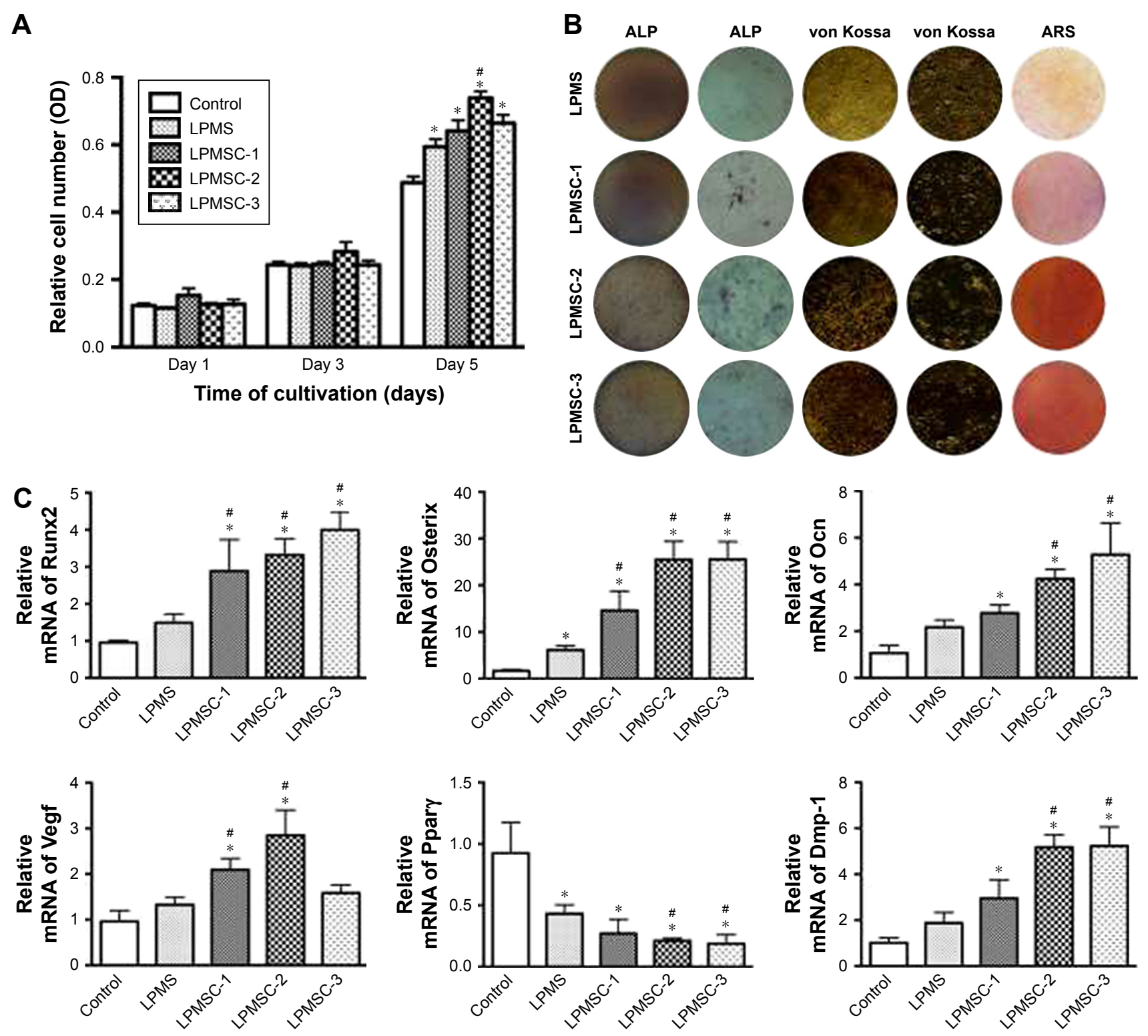

Figure 3 Proliferation and osteogenic differentiation of DPCs seeded on scaffolds. Proliferation of DPCs seeded on scaffolds different samples (A). ALP, von Kossa, and ARS staining photographs of DPCs seeded on scaffolds (B). Gene expression of Runx2, Osterix, Ocn, Vegf, Ppary, and Dmp-I for DPCs seeded on different samples after 7 days as determined by real-time PCR analysis $(\mathbf{C}) .{ }^{*} p<0.05$ vs control; ${ }^{*} p<0.05$ vs LPMS.

Abbreviations: ALP, alkaline phosphatase; ARS, alizarin red S; DPCs, dental pulp cells; LPMS, large-pore mesoporous silica; LPMSC, large-pore mesoporous Ca-Si-based bioceramics.

expression was significantly increased for cells seeded on LPMSC-1 and LPMSC-2 scaffolds compared with DPCs cultured in DMEM or seeded on LPMS scaffolds. In contrast, Ppary expression was decreased for cells seeded on both LPMS and LPMSCs.

\section{Osteogenic differentiation of DPCs} cultured in biomaterial extracts

Figure $4 \mathrm{~A}-\mathrm{C}$ indicates that the osteogenic differentiation of DPCs cultured in the control and LPMS extract was significantly lower than that of DPCs cultured in LPMSC-2 and LPMSC-3 extracts; however, there were no significant differences between the control group and LPMS group; the trend was similar to that of cells seeded on scaffolds. Compared with the control group and LPMS group, the Osterix, Bmp-2, and Dmp-1 expressions were increased in the LPMSC groups (Figure 4D). Moreover, the Opg expression and the Opg/Rankl ratio were significantly increased for cells cultured in the LPMSC-2 and LPMSC-3 extracts, which suggested that LPMSC-2 and LPMSC-3 extracts enhanced the osteogenic differentiation of DPCs. Interestingly, we observed decreased gene expression (CaSR, Bmp-2, 

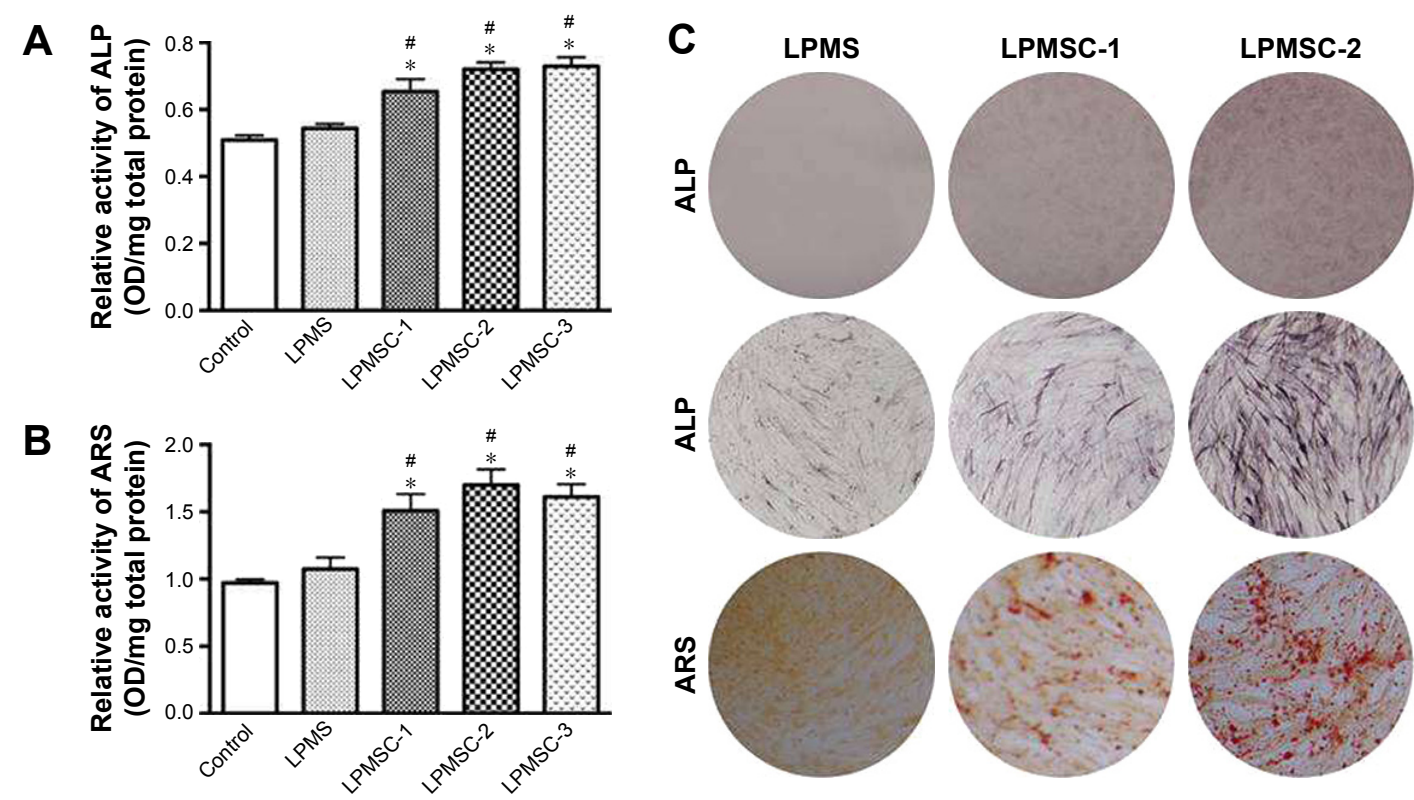

LPMSC-3

D
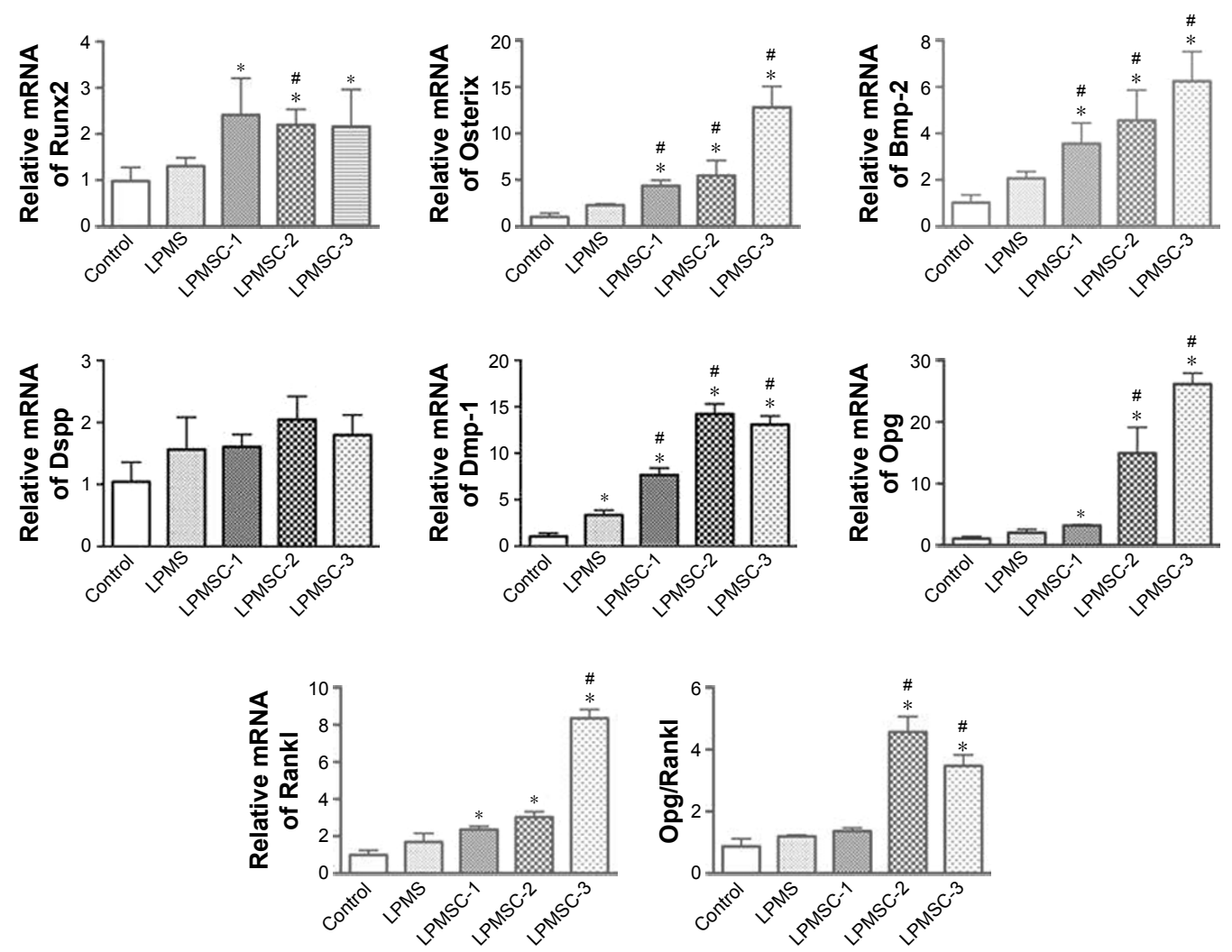

Figure 4 Osteogenic differentiation of DPCs cultured in biomaterial extracts. ALP activity (A) and mineral deposition (B) of DPCs cultured in biomaterial extracts. ALP and ARS staining photographs of DPCs cultured in biomaterial extracts (C). Gene expression of Runx2, Osterix, Bmp-2, Dspp, Dmp-I, Opg, and Rankl for DPCs cultured in different extracts after 7 days as determined by real-time PCR analysis (D). ${ }^{*} p<0.05$ vs control; ${ }^{*} p<0.05$ vs LPMS.

Abbreviations: ALP, alkaline phosphatase; ARS, alizarin red S; DPCs, dental pulp cells; LPMS, large-pore mesoporous silica; LPMSC, large-pore mesoporous Ca-Si-based bioceramics.

Alp, and Ocn) after NPS2143 (CaSR signaling pathway inhibitor) treatment of cells cultured in the LPMSC extracts (Figure 5). These results suggested that the CaSR pathway may be involved in LPMSC-stimulated cell differentiation.

\section{rhPDGF-BB release kinetics}

Figure 6A indicates that the adsorption capacity of rhPDGF-BB decreased from 13.7 to $4.6 \mathrm{mg} / \mathrm{g}$ with an increase in Ca content. Figure 6B shows the rhPDGF-BB release curve of the 

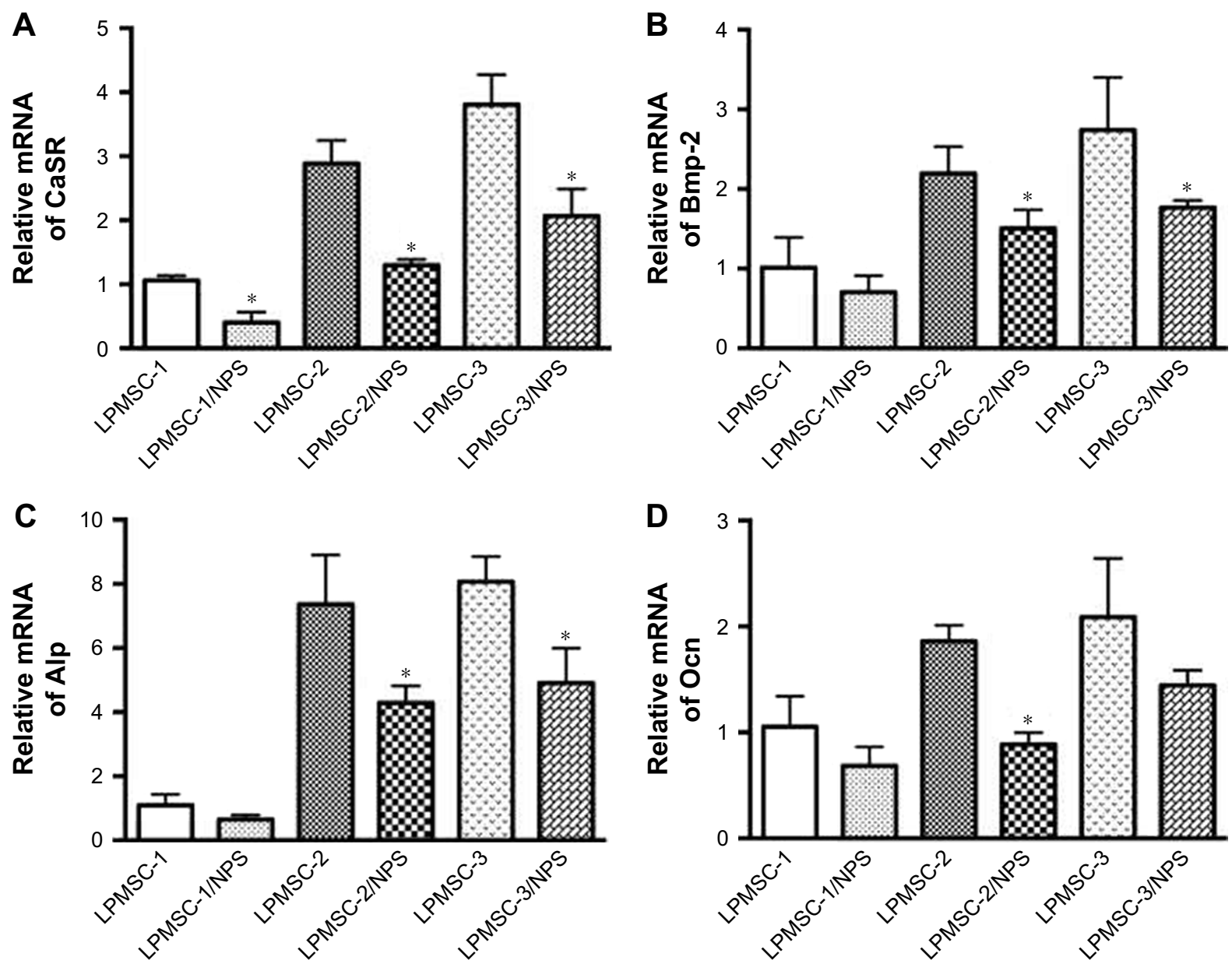

Figure 5 The gene expression of CaSR (A), Bmp-2 (B), Alp (C), and Ocn (D) for DPCs cultured in LPMSC extracts with NPS2 I 43 after 7 days. *p<0.05 vs LPMSCs. Abbreviations: DPCs, dental pulp cells; LPMS, large-pore mesoporous silica; LPMSC, large-pore mesoporous Ca-Si-based bioceramics.

scaffold. It could be observed that the release patterns of LPMSC-1 and LPMSC-2 were consistent, and they were slowly released throughout the process. However, $40 \%$ of the rhPDGF-BB was released from LPMSC-3 at 12 hours, and the release rate slowed down after 48 hours. The results suggested that the release of rhPDGF-BB from LPMS, LPMSC-1, and LPMSC-2 was slower than that from LPMSC-3 in the early stage.
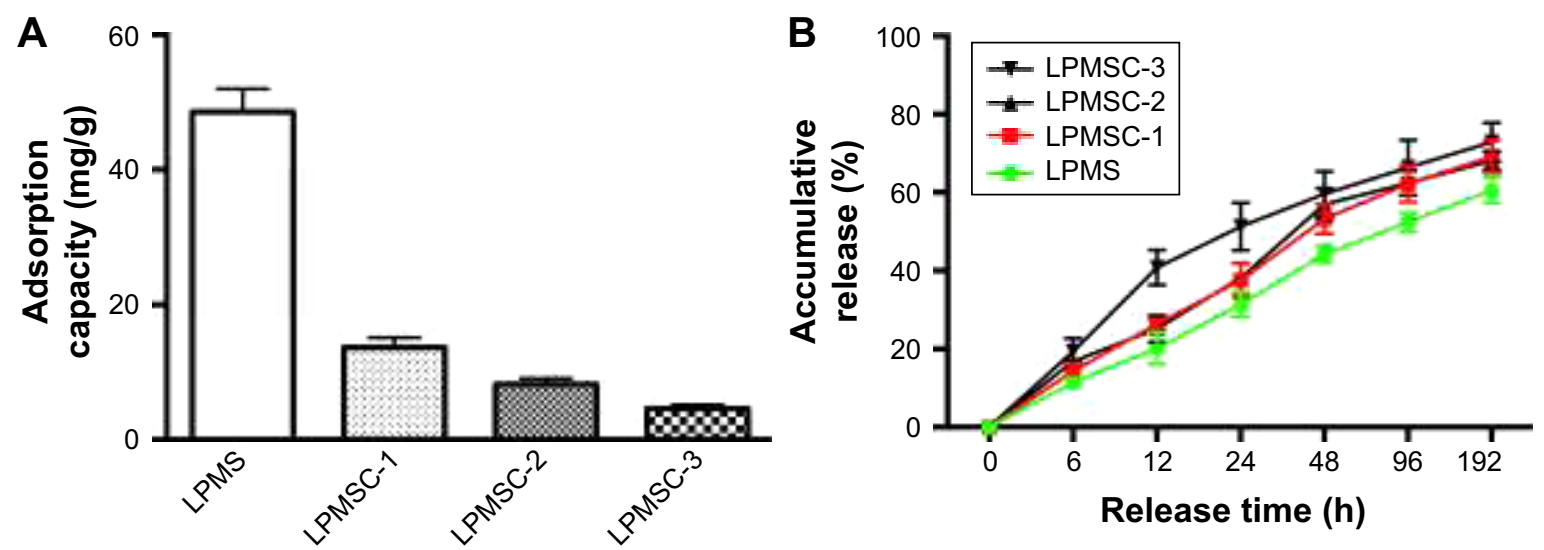

Figure 6 Adsorption capacity (A) and release performance (B) of rhPDGF-BB from LPMS and LPMSCs.

Abbreviations: LPMS, large-pore mesoporous silica; LPMSC, large-pore mesoporous Ca-Si-based bioceramics; rhPDGF-BB, recombinant human platelet-derived growth factor-BB. 


\section{Histological and histomorphometric findings}

Considering that the bone regeneration scaffold is degraded in vivo for a long time, it is necessary to evaluate its biological safety. Figure 7A reveals that there were no obvious pathological changes in the organs, including the kidney, liver, spleen, and lung. New bone formation was observed by H\&E and van Gieson's picro-fuchsin staining. The areas of new bone formation accounted for $11.54 \% \pm 1.81 \%, 18.28 \% \pm 3.54 \%$, and $17.54 \% \pm 1.20 \%$ in the LPMSC-1, LPMSC-2, and LPMSC-3 groups (Figure 7B-D), respectively, which was more than that in LPMS group $(5.19 \% \pm 2.12 \% ; p<0.05)$. Moreover, the areas of new bone formation in LPMSC-2 and LPMSC-3 groups were more than that in LPMSC-1 group.

\section{Discussion}

Regenerative medicine seeks to provide adequate biomaterials for tissue regeneration or repair. In this study, a simple and effective method was used to fabricate LPMSCs prepared using LPMS as both the silicon source and template and using $\mathrm{Ca}\left(\mathrm{NO}_{3}\right)_{2}$ as the calcium source. In addition to the large mesopore size $(22.3 \mathrm{~nm})$, the other important features of LPMS were that the organic template was removed and $\mathrm{Si}-\mathrm{OH}$ groups were maintained in the synthesis process. Moreover, the prepared LPMSCs also possess a large mesoporous diameter $(>15 \mathrm{~nm})$. According to the solid-phase reaction equation $\left(\mathrm{SiO}_{2}+\mathrm{CaO}=\mathrm{CaSiO}_{3}\right)$, when the molar ratio of $\mathrm{Si}$ to $\mathrm{Ca}$ is $1: 1$, the amorphous $\mathrm{SiO}_{2}$ is all transformed into amorphous $\mathrm{CaSiO}_{3}$. Therefore, LPMSC-3 ( $\mathrm{Si} / \mathrm{Ca}=1: 1)$ was amorphous $\mathrm{CaSiO}_{3}$, and LPMSC-1 $(\mathrm{Si} / \mathrm{Ca}=4: 1)$ and LPMSC-2 $(\mathrm{Si} / \mathrm{Ca}=2: 1)$ were the complex of amorphous $\mathrm{SiO}_{2}$ and amorphous $\mathrm{CaSiO}_{3}$.

For LPMSC-1 and LPMSC-2, partially amorphous $\mathrm{SiO}_{2}$ was transformed into amorphous $\mathrm{CaSiO}_{3}$, and the residual $\mathrm{SiO}_{2}$ retained part of the $\mathrm{Si}-\mathrm{OH}$ on the pore surface. Therefore, LPMSC-1 and LPMSC-2 could bind rhPDGF-BB via hydrogen bonds. ${ }^{26-29}$ However, there was no $\mathrm{Si}-\mathrm{OH}$ on the surface because LPMSC-3 was completely transformed
A

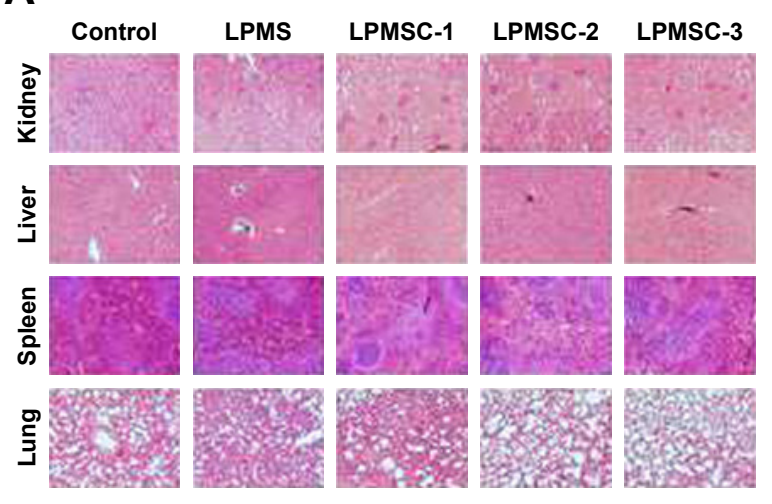

C

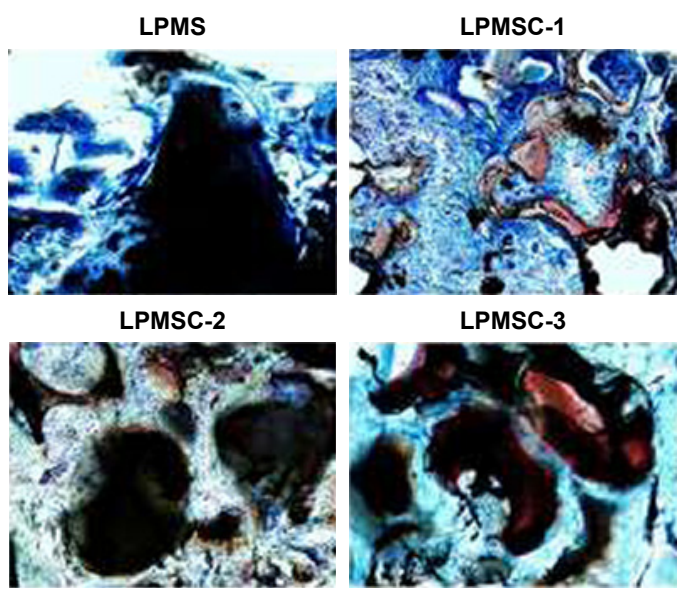

B

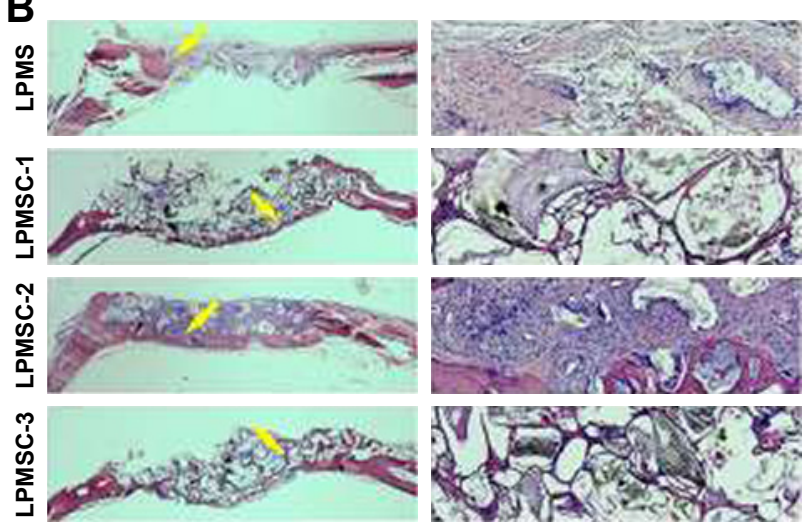

D

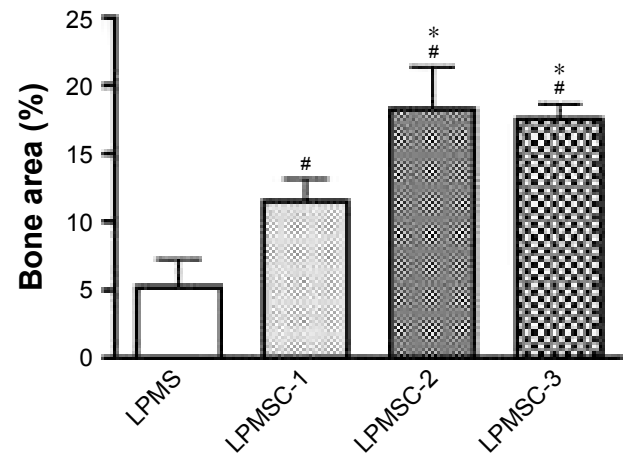

Figure 7 Histological and histomorphometric observations 8 weeks after the operation. Pathological changes in the kidney, liver, spleen, and lung (A). Different samples were stained with H\&E (yellow arrow: new bone) $(\mathbf{B})$ and van Gieson's picro-fuchsin (C). Histomorphometric analysis of the new bone area $(\mathbf{D})$. $\# p<0.05$ vs LPMS; ${ }^{*} p<0.05$ vs LPMSC-I.

Abbreviations: LPMS, large-pore mesoporous silica; LPMSC, large-pore mesoporous Ca-Si-based bioceramics. 
into amorphous $\mathrm{CaSiO} 3$, so that rhPDGF-BB could only be combined with LPMSC-3 by van der Waals force. As we all know, the van der Waals force is weaker than the hydrogen bond. It could be seen that the LPMSC-1 and LPMSC-2 that retained $\mathrm{Si}-\mathrm{OH}$ showed a better release effect (Figure 6). The pore sizes of LPMSC-1 (15.7 nm) and LPMSC-2 (15.8 nm), calculated by isothermal adsorption branches, were similar. However, the pore size of LPMSC-3 is relatively large (21.3 nm), which may be due to the excessive amount of $\mathrm{Ca}$ added and resulted in the destruction of the mesoporous pore. Although the load of rhPDGF-BB was reduced from $13.7 \mathrm{mg} / \mathrm{g}$ to $4.6 \mathrm{mg} / \mathrm{g}$, the load capacity of LPMSCs was enough because the bone regeneration required a low dose of growth factor. ${ }^{25,30}$ It is well known that not all factor release systems play a role in bone regeneration. Only the slow release of growth factors could achieve a long-term and effective promotion. ${ }^{31}$ Thus, LPMSC-1 and LPMSC-2 were more suitable as factor delivery systems compared with LPMSC-3.

Figure 3A demonstrates the enhanced cell metabolic activities in LPMS and LPMSCs groups on day 7 compared with the control group. It has been reported that the release of trace amounts of silicon from bone material does not cause osteoblast damage, whereas the trace amounts of silicates may induce osteoblast proliferation. Studies have shown that $\mathrm{Ca}$ ions also could promote cell proliferation; therefore, cell metabolic activities were enhanced with the addition of calcium content in LPMSC. Although the Ca content of LPMSC-3 is higher than that of LPMSC-2, the effect of promoting cell metabolic activity was slightly worse compared with LPMSC-2, which may be related to the change in surface topology/roughness and the $\mathrm{pH}$ value of solution. LPMSC-3 was amorphous $\mathrm{CaSiO}_{3}$, and LPMSC-1 and LPMSC-2 were the complex of amorphous $\mathrm{SiO}_{2}$ and amorphous $\mathrm{CaSiO}_{3}$. Moreover, due to the high $\mathrm{Ca}$ content of LPMSC-3, the faster the ion exchange rate in the liquid, the higher the $\mathrm{pH}$ value of the solution.

Furthermore, the ALP activity and key osteogenic transcription genes (Runx2 and Osterix) in LPMSC groups were higher than those of the LPMS group (Figure 3B and C). Moreover, the ALP activity and semi-quantitative ARS mineralization of DPCs cultured in LPMS extracts were also significantly lower than those of DPCs cultured in LPMSC extracts (Figure 4A-C). However, there were no significant differences between the differentiation of DPCs in the control group and LPMS group. Figure $3 \mathrm{C}$ reveals that LPMSC-2 and LPMSC-3 can promote the expression of these three osteogenic-related markers (Osterix, Ocn, and
Opg/Rankl), which suggested that the materials with the high calcium content (LPMSC-2 and LPMSC-3) were stronger than calcium-free material (LPMS) or materials with low calcium content (LPMSC-1). Compared with LPMS group, Osterix, Bmp-2, and Dmp-1 expressions were increased in the LPMSC groups (Figure 4C). These results indicated that the DPCs seeded on LPMSCs were more likely to enhance osteogenic differentiation.

Interestingly, we observed increased CaSR expression of DPCs cultured in LPMSC-2 extracts and decreased gene expression (Bmp-2, Alp, and Ocn) after NPS2143 (CaSR signaling pathway inhibitor) treatment of cells cultured in LPMSC-2 extracts (Figure 5). CaSR is a member of the G-protein-coupled receptor family located on chromosome 3q13.3-21 and has been considered an appealing target for osteoporosis. ${ }^{32,33}$ Both the early and late osteogenic genes were reduced in CaSR knockout mice, ${ }^{34}$ resulting in decreased cortical and trabecular bone masses. These results suggest that LPMSCs possess favorable properties for the osteogenic differentiation of DPCs via the CaSR signaling pathway.

\section{Conclusion}

The present study demonstrated that LPMSCs promoted the osteogenic differentiation of DPCs through the CaSR pathway, especially the LPMSC-2 and LPMSC-3 with higher calcium content. Furthermore, the loading capacity for rhPDGF-BB was satisfactory in LPMSCs. For the calvarial defect repair, the areas of new bone formation were increased in the LPMSC-2 and LPMSC-3 groups compared with the LPMSC-1 and LPMS groups. We concluded that LPMSC-2 and LPMSC-3 possessed both excellent osteogenic abilities and satisfactory loading capacities, which may be attributed to their moderate $\mathrm{Ca} / \mathrm{Si}$ molar ratio. Therefore, LPMSCs with moderate $\mathrm{Ca} / \mathrm{Si}$ molar ratio might be potential alterative grafts for craniomaxillofacial bone regeneration.

\section{Acknowledgments}

Joint financial support from the National Key Research and Development Program of China (2016YFC1102900), the National Natural Science Foundation of China (81300853, 81430012, 81620108006, 31500787, and 5141101086), the Science and Technology Commission of Shanghai Municipality (15XD1502500 and 15410722700), and the Medicine and Engineering Cross Research Foundation of Shanghai Jiao Tong University (YG2015ZD06) is acknowledged.

\section{Disclosure}

The authors report no conflicts of interest in this work. 


\section{References}

1. Tang F, Li L, Chen D. Mesoporous silica nanoparticles: synthesis, biocompatibility and drug delivery. Adv Mater. 2012;24(12):1504-1534.

2. Wang Y, Zhao Q, Han N, et al. Mesoporous silica nanoparticles in drug delivery and biomedical applications. Nanomedicine. 2015; 11(2):313-327.

3. Fang X, Zhao X, Fang W, Chen C, Zheng N. Self-templating synthesis of hollow mesoporous silica and their applications in catalysis and drug delivery. Nanoscale. 2013;5(6):2205-2218.

4. El-Boubbou K, Schofield DA, Landry CC. Enhanced enzymatic thermal stability and activity in functionalized mesoporous silica monitored by (31) p NMR. Adv Healthc Mater. 2012;1(2):183-188.

5. Zhang W, Zhu C, Wu Y, et al. VEGF and BMP-2 promote bone regeneration by facilitating bone marrow stem cell homing and differentiation. Eur Cell Mater. 2014;27:1-11.

6. Linkhart TA, Mohan S, Baylink DJ. Growth factors for bone growth and repair: IGF, TGF beta and BMP. Bone. 1996;19(1 Suppl):1S-12S.

7. Friedlaender GE, Lin S, Solchaga LA, Snel LB, Lynch SE. The role of recombinant human platelet-derived growth factor-BB (rhPDGF-BB) in orthopaedic bone repair and regeneration. Curr Pharm Des. 2013; 19(19):3384-3390.

8. Scheufler C, Sebald W, Hülsmeyer M. Crystal structure of human bone morphogenetic protein-2 at $2.7 \AA$ resolution. J Mol Biol. 1999;287(1): $103-115$.

9. Kuai J, Mosyak L, Brooks J, et al. Characterization of binding mode of action of a blocking anti-platelet-derived growth factor (PDGF)-B monoclonal antibody, MOR8457, reveals conformational flexibility and avidity needed for PDGF-BB to bind PDGF receptor- $\beta$. Biochemistry. 2015;54(10):1918-1929.

10. Iyer S, Scotney PD, Nash AD, Ravi Acharya K. Crystal structure of human vascular endothelial growth factor-B: identification of amino acids important for receptor binding. J Mol Biol. 2006;359(1):76-85.

11. Schmidt-WinkelP, Lukens WW, Zhao D, Yang P, Chmelka BF, Stucky GD. Mesocellular siliceous foams with uniformly sized cells and windows. J Am Chem Soc. 1999;121(1):254-255.

12. Zhao D, Huo Q, Feng J, Chmelka BF, Stucky GD. Nonionic triblock and star diblock copolymer and oligomeric surfactant syntheses of highly ordered, hydrothermally stable, mesoporous silica structures. J Am Chem Soc. 1998;120(24):6024-6036.

13. Margolese D, Melero JA, Christiansen SC, Chmelka BF, Stucky GD. Direct syntheses of ordered SBA-15 mesoporous silica containing sulfonic acid groups. Chem Mater. 2000;12(8):2448-2459.

14. Hench LL, Splinter RJ, Allen WC, Greenlee TK. Bonding mechanisms at the interface of ceramic prosthetic materials. J Biomed Mater Res. 1971;5(6):117-141.

15. Hench LL, Paschall HA. Histochemical responses at a biomaterial's interface. J Biomed Mater Res. 1974;8(3):49-64.

16. Hench LL. Physical chemistry of glass surfaces. J Non-Cryst Solids. 1977;28(1):83-105.

17. Capiod T. Cell proliferation, calcium influx and calcium channels. Biochimie. 2011;93(12):2075-2079.

18. Tiwari M, Prasad S, Shrivastav TG, Chaube SK. Calcium signaling during meiotic cell cycle regulation and apoptosis in mammalian oocytes. J Cell Physiol. 2017;232(5):976-981.
19. Hennings H, Michael D, Cheng C, Steinert P, Holbrook K, Yuspa SH. Calcium regulation of growth and differentiation of mouse epidermal cells in culture. Cell. 1980;19(1):245-254.

20. Tonelli FM, Santos AK, Gomes DA, et al. Stem cells and calcium signaling. Adv Exp Med Biol. 2012;740:891-916.

21. Cheng S, Wang W, Lin Z, et al. Effects of extracellular calcium on viability and osteogenic differentiation of bone marrow stromal cells in vitro. Hum Cell. 2013;26(3):114-120.

22. Zhang XD, Zeng DL, Li N, Jiang X, Liu C, Li Y. Large-pore mesoporous $\mathrm{Ca}-\mathrm{Si}$-based bioceramics with high in vitro bioactivity and protein adsorption capability for bone tissue regeneration. J Mater Chem B. 2016;4(22):3916-3924.

23. Zeng D, Xia L, Zhang W, et al. Maxillary sinus floor elevation using a tissue-engineered bone with calcium-magnesium phosphate cement and bone marrow stromal cells in rabbits. Tissue Eng Part A. 2012;18: $870-881$.

24. Zhang X, Zeng D, Li N, et al. Functionalized mesoporous bioactive glass scaffolds for enhanced bone tissue regeneration. Sci Rep. 2016;6:19361.

25. Xu L, Lv K, Zhang W, Zhang X, Jiang X, Zhang F. The healing of critical-size calvarial bone defects in rat with rhPDGF-BB, BMSCs, and $\beta$-TCP scaffolds. J Mater Sci Mater Med. 2012;23(4):1073-1084.

26. Shylesh S, Hanna D, Gomes J, Canlas CG, Head-Gordon M, Bell AT. The role of hydroxyl group acidity on the activity of silica-supported secondary amines for the self-condensation of n-butanal. ChemSusChem. 2015;8(3):466-472.

27. Wu XL, Xiong SJ, Zhu J, Wang J, Shen JC, Chu PK. Identification of surface structures on $3 \mathrm{C}$-SiC nanocrystals with hydrogen and hydroxyl bonding by photoluminescence. Nano Lett. 2009;9(12):4053-4060.

28. Hou D, Li Z, Zhao T, Zhang P. Water transport in the nano-pore of the calcium silicate phase: reactivity, structure and dynamics. Phys Chem Chem Phys. 2015;17(2):1411-1423.

29. Zhang J, Zhou HJ, Yang K, Yuan Y, Liu CS. RhBMP-2-loaded calcium silicate/calcium phosphate cement scaffold with hierarchically porous structure for enhanced bone tissue regeneration. Biomaterials. 2013;34(37):9381-9392.

30. Zhang W, Jin YQ, Qian S, et al. Vacuum extraction enhances rhPDGF-BB immobilization on nanotubes to improve implant osseointegration in ovariectomized rats. Nanomedicine. 2014;10(8):1809-1818.

31. Zhao J, Shen G, Liu C, et al. Enhanced healing of rat calvarial defects with sulfated chitosan-coated calcium-deficient hydroxyapatite/bone morphogenetic protein 2 scaffolds. Tissue Eng Part A. 2012;18(1-2): 185-197.

32. Lane NE, Sanchez S, Modin G, Genant HK, Pierini E, Arnaud CD. Parathyroid hormone treatment can reverse corticosteroid-induced osteoporosis. results of a randomized controlled clinical trial. J Clin Invest. 1998;102:1627-1633.

33. Marie PJ. The calcium-sensing receptor in bone cells: a potential therapeutic target in osteoporosis. Bone. 2010;46:571-576.

34. Trindade R, Albrektsson T, Tengvall P, Wennerberg A. Foreign body reaction to biomaterials: on mechanisms for buildup and breakdown of osseointegration. Clin Implant Dent Relat Res. 2016;18:192-203.
International Journal of Nanomedicine

\section{Publish your work in this journal}

The International Journal of Nanomedicine is an international, peerreviewed journal focusing on the application of nanotechnology in diagnostics, therapeutics, and drug delivery systems throughout the biomedical field. This journal is indexed on PubMed Central,

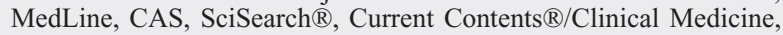

\section{Dovepress}

Journal Citation Reports/Science Edition, EMBase, Scopus and the Elsevier Bibliographic databases. The manuscript management system is completely online and includes a very quick and fair peer-review system, which is all easy to use. Visit http://www.dovepress.com/ testimonials.php to read real quotes from published authors. 